\title{
SECRET domain of variola virus CrmB protein can be a member of poxviral type II chemokine- binding proteins family
}

\author{
Denis V Antonets*', Tatyana S Nepomnyashchikh, Sergei N Shchelkunov
}

\begin{abstract}
Background: Variola virus (VARV) the causative agent of smallpox, eradicated in 1980, have wide spectrum of immunomodulatory proteins to evade host immunity. Recently additional biological activity was discovered for VARV CrmB protein, known to bind and inhibit tumour necrosis factor (TNF) through its $\mathrm{N}$-terminal domain homologous to cellular TNF receptors. Besides binding TNF, this protein was also shown to bind with high affinity several chemokines which recruit B- and T-lymphocytes and dendritic cells to sites of viral entry and replication. Ability to bind chemokines was shown to be associated with unique C-terminal domain of CrmB protein. This domain named SECRET (Smallpox virus-Encoded Chemokine Receptor) is unrelated to the host proteins and lacks significant homology with other known viral chemokine-binding proteins or any other known protein.

Findings: De novo modelling of VARV-CrmB SECRET domain spatial structure revealed its apparent structural homology with cowpox virus CC-chemokine binding protein ( $\mathrm{VCCl}$ ) and vaccinia virus A41 protein, despite low sequence identity between these three proteins. Potential ligand-binding surface of modelled VARV-CrmB SECRET domain was also predicted to bear prominent electronegative charge which is characteristic to known orthopoxviral chemokine-binding proteins.

Conclusions: Our results suggest that SECRET should be included into the family of poxviral type II chemokinebinding proteins and that it might have been evolved from the vCCl-like predecessor protein.
\end{abstract}

\section{Introduction}

Chemokines form a large family of chemoattractant cytokines with low molecular weight ( 7-14 kDa) and their sequence similarity range from 20 to $90 \%$. More than 50 distinct chemokines and over 19 different chemokine receptors were discovered to date. All known cellular chemokine receptors are type III transmembrane proteins associated with G-proteins. According to the arrangement of conservative $\mathrm{N}$-terminal cysteine residues chemokines are divided into $\mathrm{CC}$-, $\mathrm{CXC}$-, $\mathrm{C}$ - and CX3C-chemokines [1]. Chemokines play important roles in regulation of innate as well as acquired immunity orchestrating leukocyte migration. Through binding to cell surface or intercellular matrix glycosaminoglycans (GAGs) chemokines form chemotactic gradient attracting leukocytes to the sites of injury and inflammation.

\footnotetext{
* Correspondence: antonec@yandex.ru

State Research Center of Virology and Biotechnology "Vector"

Rospotrebnadzor, Novosibirsk region, Koltsovo, Russian Federation
}

Chemokines are also involved in embryonic development, organogenesis and other processes $[1,2]$. Despite low sequence identity between different chemokines all of them share remarkably similar tertiary structure with elongated $\mathrm{N}$-terminal loop followed by three $\beta$-chains folded in a "greek key" moiety [3].

During long-lasting coevolution with their hosts poxviruses have developed efficient mechanisms to evade host immune reactions initiated in response to infection. In particular, one of the most important viral immunomodulatory strategies are viroceptors - virally encoded proteins secreted from infected cells - which bind and inhibit biological activity of tumour necrosis factor (TNF), different kinds of interferons, interleukine 18, chemokines and other mediators of host immune system [4-6]. Such viroceptors may be non-homologous to cellular proteins with correspondent biological activity, as it was shown for poxviral chemokine-binding proteins [7]. Some poxviral immunomodulatory proteins possess 
pleiotropic activity, as it was shown for M-T7 protein of myxoma virus, which is able to bind $\gamma$ IFN as well as chemokines [8]. Recently additional biological activity was discovered for orthopoxviral $\mathrm{CrmB}$ and $\mathrm{CrmD}$ proteins. Previously known only as TNF-binding proteins, they were shown to bind with high affinity and inhibit CCL25, CCL28, CCL25, CXCL12 $\beta$, CXCL13 and CXCL14 chemokines. Chemokine-binding activity of $\mathrm{CrmB}$ and CrmD proteins was shown to be mediated by their unique C-terminal domain lacking substantial homology to any other known proteins. This domain was named SECRET - Smallpox virus-Encoded Chemokine Receptor [9].

Since C-terminal domain of VARV-CrmB lacks substantial sequence homology with other proteins (excepting orthologous proteins of other orthopoxviruses), and since its spatial structure is not known, we decided to predict it to find related proteins sharing similar structural features with VARV-CrmB SECRET domain. Predicted spatial structure of VARV CrmB SECRET domain was found to be closely related to cowpox virus vCCI protein, belonging to the family of poxviral type II chemokine-binding proteins.

\section{Results and Discussion}

Using PSIPRED server [10] VARV-CrmB SECRET domain was predicted to consist of twelve $\beta$-strands linked by unordered loops, remaining the overall secondary structure of poxviral type II chemokine-binding proteins (vCkBPII). Using I-TASSER web-server [11], which was shown to be the best server for predicting spatial structures of proteins according to results of CASP7 (CASP - Critical Assessment of protein Structure Prediction) and CASP8 competitions [11,12], we obtained the model of VARV-CrmB SECRET domain. SECRET was predicted to be $\beta$-sandwich, composed by two parallel $\beta$-sheets connected by several loops (Figure $1)$. The exterior surface of the first $\beta$-sheet was hidden from the solvent by two long engirdling loops. The I-TASSER output can be found in Additional file 1 .

As the best template for modelling SECRET spatial structure I-TASSER server has chosen the structure of CPXV vCCI protein [PDB: 1CQ3] belonging to the family of poxviral type II chemokine-binding proteins which are encoded by almost all known members of Orthopoxvirus and Leporipoxvirus genera. These abundantly secreted during the early stages of infection 35 $\mathrm{kDa}$ glycoproteins lack any homology to cellular chemokine receptors as well as to other known proteins $[3,13]$. This protein family also includes VACV A41 protein and its orthologs [14]. Despite low sequence identity ( 20\%) VACV A41 [PDB:2VGA] and CPXV vCCI [PDB:1CQ3] proteins share remarkably similar tertiary structure [14]. We calculated superposition of vCCI and

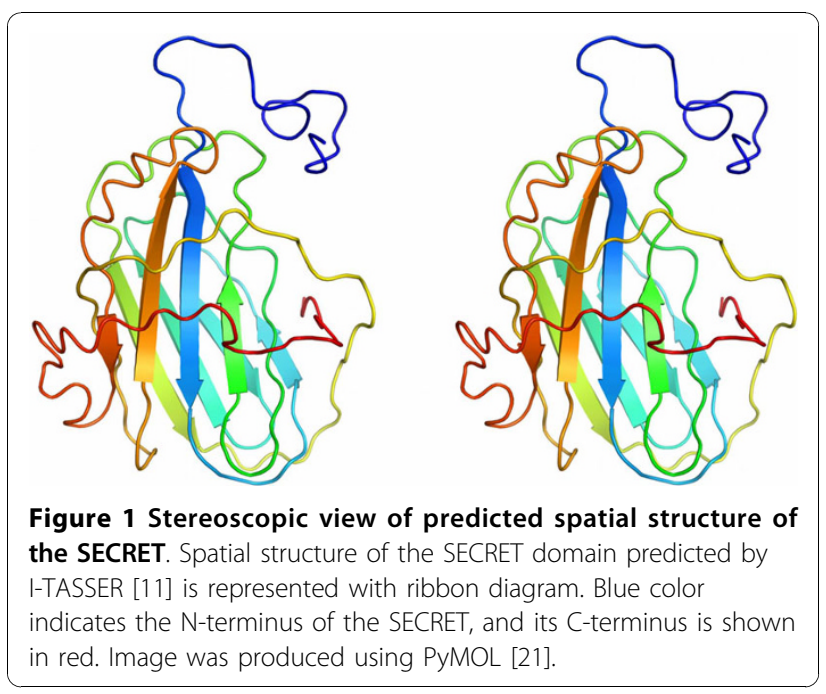

A41 spatial structures with predicted tertiary structure of the SECRET domain (Figure 2A). Superpositions were made using CEalign [15]. Root mean square deviation (RMSD) between coordinates of aligned $\mathrm{C} \alpha$ atoms of SECRET and vCCI was about $2.33 \AA$ (identity between structurally aligned amino acid residues was 14.2\%), RMSD between coordinates of aligned $\mathrm{C} \alpha$ atoms of SECRET and A41 was $3.28 \AA$ (10.8\% of identity), RMSD between coordinates of aligned $\mathrm{C} \alpha$ atoms of A41 and vCCI was about $3.06 \AA$ (19.9\% of identity). The sequences of these proteins were aligned using TMalign [16] server and then manually edited according to their either predicted (SECRET) or known (vCCI, A41) secondary structures (Figure 2B). According to produced alignment SECRET shared $16.3 \%$ of sequence identity with vCCI and $15.3 \%$ with A41 protein; sequence identity of vCCI to A41 was about $25.5 \%$.

Although spatial structures of vCkBPII proteins are remarkably similar, different members of the family have notable characteristic structural differences. Thus VACV A41 lacks long extended loop which connects the $2^{\text {nd }}$ and the $3^{\mathrm{d}} \beta$ strands of vCCI (Figure 2A). This loop is conserved in vCCI and its orthologs and was shown to make these proteins able to bind wide spectrum of CC-chemokines with high affinity $[3,13,17]$. Comparison of known tertiary structure of VACV A41 with predicted spatial structure of the SECRET domain of VARV CrmB revealed that SECRET probably also lacks this loop (Figure 2). This finding gives a bit of support to hypothesis of Ruiz-Arguello and his colleagues [14] that high selectivity and similar specificity towards chemokines, observed for VACV A41 and ectromelia virus (ECTV) E163 protein (orthologous to A41) and SECRET-domain containing proteins, should be underlaid by common structural traits of these vCkBPs. VACV A41 and ECTV E163 bind with high affinity 


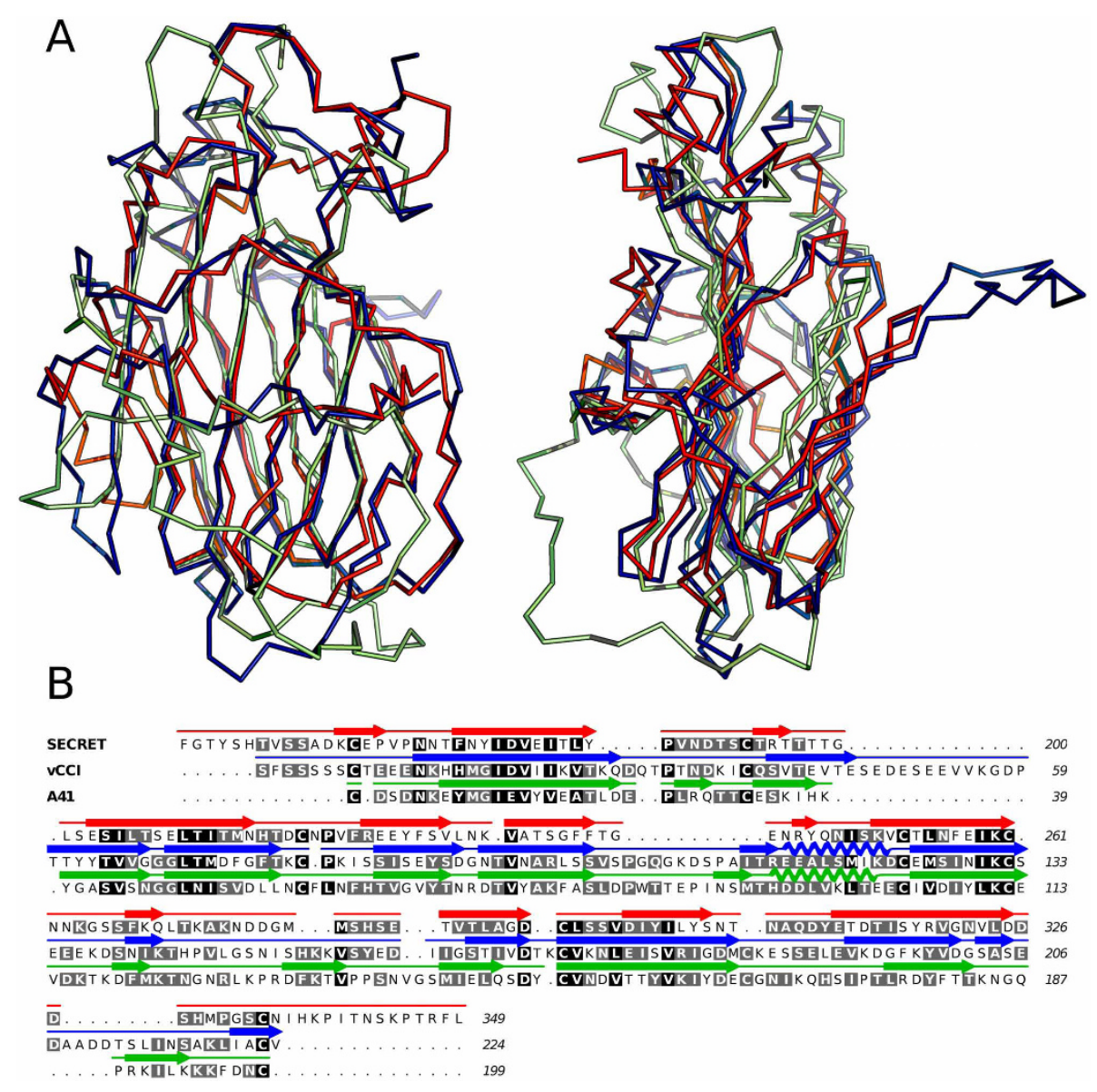

Figure 2 Superposition of vCCI and A41 structures with modelled SECRET and alignment of their sequences. (A) Superposition of $\mathrm{VCCl}$, A41 and SECRET was made with CEalign [15]. SECRET is red coloured, $\mathrm{vCCl}$ is shown in blue and A41 is green. Left panel shows surfaces formed by $\beta$-sheets I of these proteins. Right panel shows the same superposition rotated around y-axis by $90^{\circ}$. Characteristic long extended loop of $\mathrm{VCCl}$ is absent in A41 as well as in the SECRET. (B) Alignment of $\mathrm{VCCl}, \mathrm{A} 41$ and SECRET amino acid sequences produced with structural alignment server TM-align [16]. Alignment was manually edited taking in mind their secondary structures (shown above corresponding sequences) using Aline software [22]. Numbering of amino acid residues in SECRET corresponds to that of full length VARV-CrmB protein (P34015). Gaps are shown as dots, sequences are coloured by amino acid similarity. Amino acid residues of VCCl and A41 are numbered according to numeration found in 1CQ3 and 2VGA PDB files, respectively.

CCL21, 25, 26, 28, CXCL12 $\alpha$, CXCL12 $\beta$, CXCL13 and CXCL14 chemokines [3,14]; and SECRET domains of VARV CrmB, ECTV CrmD and some other SECRETcontaining proteins were shown to bind with high affinity CCL25, 28, CXCL12 $\beta$, CXCL13 and CXCL14 chemokines [9].

Furthermore, although in all known vCkBPs exterior surface of the second $\beta$ sheet has pronounced electronegative potential due to multiple acidic amino acid residues (Figure 3A) that is favourable to the interaction with positively charged conservative patches of basic amino acid residues found in all known chemokines $[3,7,13,17]$, charge distributions of the opposite surfaces of different $\mathrm{vCkBPs}$ may have remarkable distinctions $[3,18]$ (Figure 3B). For example, in vCCI and in the majority of its orthologs this surface has weak electrostatic charge, whereas corresponding surfaces of A41 and its orthologs have pronounced positive charge making these proteins able to bind negatively charged glycosaminoglycans (GAGs) [3]. Myxoma virus M-T1 protein was also shown to bind GAGs, and unlike the majority of vCCI orthologs it has prominent positive electrostatic potential on the surface formed by $\beta$-sheet I encircled by two loops [18]. Thus ability to bind GAGs makes certain vCkBPs capable of blocking leukocyte influx into the site of viral replication interfering with chemotactic gradient formation through inhibiting interaction of chemokines with cell surface and extracellular matrix GAGs $[3,18,19]$. Other vCkBPs, which could not bind GAGs, realize their biological activity only through competing with cellular chemokine receptors for chemokines binding. As predicted by us VARV 


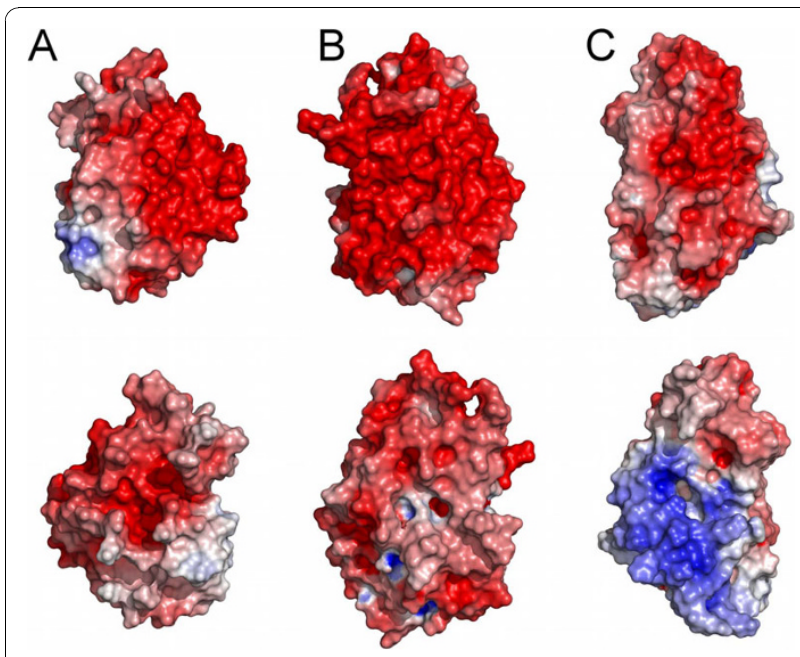

Figure 3 Electrostatic potential surfaces of SECRET (A), vCCI (B) and A41 (C). Upper panel shows chemokine-binding surfaces formed by $\beta$-sheets II of corresponding molecules. Lower panel shows the same molecules rotated around $y$-axis by $180^{\circ}$. Electrostatic potential was calculated with DelPhi software [20]. Negatively charged area is coloured in red and blue colour indicates positive electrostatic charge. Image was produced using PyMOL $[21]$

CrmB SECRET domain also has prominent electronegative potential on the surface formed by $\beta$-sheet II, but its opposite surface have no positively charged areas (Figure 3). Thus VARV CrmB SECRET domain is likely unable to bind GAGs and should realize its biological activity only through its ability to interfere with binding of certain chemokines to their cellular receptors that was discovered by Alejo and his colleagues [9].

\section{Conclusions}

Our prediction that VARV CrmB SECRET domain belongs to the family of poxviral type II chemokinebinding proteins is supported by the following: 1 . SECRET was shown to bind several chemokines with high affinity [9]; 2. its ligand-binding surface was predicted to have prominent electronegative charge required for binding to positively charged conservative amino-acid residues of chemokines, as it was shown for other members of vCkBPsII $[3,7,13,17] ; 3$. its selectivity towards chemokines is likely to be associated with lacking long extended negatively charged loop (present in vCCI and its orthologs $[3,7,13,17]$ ) as it was shown for A41 protein [3]. Thus the predicted structural similarity of SECRET with other vCkBPsII indicates that despite low similarity of their sequences they were most likely derived from the common ancestor. It's curiously that in genomes of Leporipoxvirus and Orthopoxvirus genera members genes coding for $\mathrm{CrmB}$ orthologs are situated in immediate proximity to the genes coding for vCCI orthologs, and we think, it would be of interest to examine phylogenetic relations between the SECRET domain of $\mathrm{CrmB}$ and vCCI orthologs.

\section{Methods}

Prediction of secondary structure of VARV-CrmB SECRET domain was done using PSIPRED server [10]. Spatial structure of SECRET domain was modeled using I-TASSER web-server [11]. The server selected the structure of cowpox virus (CPXV) vCCI (viral CC-chemokine inhibitor) protein [PDB:1CQ3] as the best template for modelling SECRET domain. Electrostatic potential of molecular surfaces of all compared proteins was calculated using DelPhi software [20]. Superpositions of the structures were made using CEalign [15]. All molecular graphic images were produced using PyMOL [21]. VARV-CrmB amino acid sequence used in this work was taken from [Swiss-Prot:P34015]. Amino acid sequences of CPXV vCCI [PDB:1CQ3], vaccinia virus (VACV) A41 chemokine-binding protein [PDB:2VGA] and VARV-CrmB SECRET domain were structurally aligned using TM-align server [16]. Alignment was manually edited taking in mind the secondary structures (shown above corresponding sequences) of the proteins using Aline software [22].

\section{Additional material}

Additional file 1: Output from I-TASSER web-server. This file contains the output from I-TASSER web-server including all generated models and alignments.

\section{Acknowledgements}

This work was supported by Russian Foundation for Basic Research (grant \#09-04-00055a).

\section{Authors' contributions}

DVA designed the concept of the study, submitted the modelling task to ITASSER, analyzed produced models and drafted the manuscript. TSN has produced molecular images and alignments. She was also involved in drafting and writing the manuscript. SNS has been involved in writing the manuscript and in its critical revision and has given the final approval for the version to be published. All authors read and approved the final manuscript.

\section{Competing interests}

The authors declare that they have no competing interests.

Received: 17 June 2010 Accepted: 27 October 2010 Published: 27 October 2010

\section{References}

1. Boomker JM, de Leij LF, The TH, Harmsen MC: Viral chemokinemodulatory proteins: tools and targets. Cytokine Growth Factor Rev 2005, 16:91-103.

2. Charo IF, Ransohoff RM: The many roles of chemokines and chemokine receptors in inflammation. $N$ Engl J Med 2006, 354:610-621. 
3. Bahar MW, Kenyon JC, Putz MM, Abrescia NG, Pease JE, Wise EL, Stuart DI Smith GL, Grimes JM: Structure and function of A41, a vaccinia virus chemokine binding protein. PLoS Pathog 2008, 4:e5.

4. Alcami A, Koszinowski UH: Viral mechanisms of immune evasion. Mol Med Today 2000, 9:365-372

5. Seet BT, Johnston JB, Brunetti CR, Barrett JW, Everett $H$, Cameron C, Sypula J, Nazarian SH, Lucas A, McFadden G: Poxviruses and immune evasion. Annu Rev Immunol 2003, 21:377-423.

6. Shchelkunov SN: Immunomodulatory proteins of orthopoxviruses. Molecular Biology (Mosk) 2003, 37:41-53.

7. Carfi A, Smith CA, Smolak PJ, McGrew J, Wiley DC: Structure of a soluble secreted chemokine inhibitor vCCl (p35) from cowpox virus. Proc Natl Acad Sci USA 1999, 96:12379-12383.

8. Lalani AS, Graham K, Mossman K, Rajarathnam K, Clark-Lewis I, Kelvin D, McFadden G: The purified myxoma virus gamma interferon receptor homolog M-T7 interacts with the heparin-binding domains of chemokines. J Virol 1997, 71:4356-4363.

9. Alejo A, Ruiz-Arguello MB, Ho Y, Smith VP, Saraiva M, Alcami A: A chemokine-binding domain in the tumor necrosis factor receptor from variola (smallpox) virus. Proc Natl Acad Sci USA 2006, 103:5995-6000.

10. Bryson K, McGuffin LJ, Marsden RL, Ward JJ, Sodhi JS, Jones DT: Protein structure prediction servers at University College London. Nucl Acids Res 2005, 33:W36-38

11. Zhang $Y$ : I-TASSER server for protein $3 D$ structure prediction. $B M C$ Bioinformatics 2008, 9:40.

12. Zhang Y: I-TASSER: fully automated protein structure prediction in CASP8. Proteins 2009, 77:100-113.

13. Arnold PL, Fremont DH: Structural determinants of chemokine binding by an Ectromelia virus-encoded decoy receptor. J Virol 2006, 80:7439-7449.

14. Ruiz-Arguello MB, Smith VP, Campanella GS, Baleux F, Arenzana-Seisdedos F, Luster AD, Alcami A: An ectromelia virus protein that interacts with chemokines through their glycosaminoglycan binding domain. J Virol 2008, 82:917-926.

15. Shindyalov IN, Bourne PE: Protein structure alignment by incremental combinatorial extension (CE) of the optimal path. Protein Eng 1998, 11:739-747.

16. Zhang $Y$, Skolnick J: TM-align: A protein structure alignment algorithm based on TM-score. Nucleic Acids Research 2005, 33:2302-2309.

17. Zhang L, Derider M, McCornack MA, Jao SC, Isern N, Ness T, Moyer R, LiWang PJ: Solution structure of the complex between poxvirus-encoded CC chemokine inhibitor vCCl and human MIP-1beta. Proc Natl Acad Sci USA 2006, 103:13985-13990.

18. Seet BT, Barrett J, Robichaud J, Shilton B, Singh R, McFadden G: Glycosaminoglycan binding properties of the myxoma virus CCchemokine inhibitor, M-T1. J Biol Chem 2001, 276:30504-30513.

19. Lalani AS, McFadden G: Evasion and exploitation of chemokines by viruses. Cytokine Growth Factor Rev 1999, 10:219-233.

20. Rocchia W, Alexov E, Honig B: Extending the Applicability of the Nonlinear Poisson-Boltzmann Equation: Multiple Dielectric Constants and Multivalent lons. J Phys Chem B 2001, 105:6507-6514.

21. DeLano WL: The PyMOL Molecular Graphics System. DeLano Scientific Palo Alto, CA, USA; 2002 [http://www.pymol.org].

22. Bond CS, Schuttelkopf AW: ALINE: a WYSIWYG protein-sequence alignment editor for publication-quality alignments. Acta Cryst 2009, 65:510-512. protein can be a member of poxviral type II chemokine-binding proteins family. BMC Research Notes 2010 3:271.

\section{Submit your next manuscript to BioMed Central and take full advantage of:}

- Convenient online submission

- Thorough peer review

- No space constraints or color figure charges

- Immediate publication on acceptance

- Inclusion in PubMed, CAS, Scopus and Google Scholar

- Research which is freely available for redistribution

Submit your manuscript at www.biomedcentral.com/submit
C Biomed Central 